GANIT J. Bangladesh Math. Soc. (ISSN 1606-3694) Vol. 33 (2013) 13 - 24

\title{
DATA DEPENDENCE FOR NOOR ITERATIVE SCHEME DEALING WITH CONTRACTIVE-LIKE OPERATORS
}

\author{
Md. Asaduzzaman ${ }^{1}$ and Md. Zulfikar Ali ${ }^{2}$ \\ ${ }^{1}$ Department of Mathematics, Islamic University, Kushtia \\ ${ }^{2}$ Department of Mathematics, University of Rajshahi, Rajshahi \\ E-mail: masad_iu_math@yahoo.com and zulfikar_math_ru@yahoo.com
}

Received 25.04.12 Accepted 30.07.13

\begin{abstract}
In this paper, we have established the convergence result and the data dependence for Noor iterative scheme dealing with contractive-like operators. A numerical example has also given, in which instead of computing the fixed point of an operator, we have approximated the operator with a contractive-like one.
\end{abstract}

Keywords: Mann iterative scheme, Ishikawa iterative scheme, Noor iterative scheme, Data Dependence, Contractive-Like Operators.

\section{Introduction}

The Mann iterative scheme, known as one-step iterative scheme [18], invented in 1953, was used to prove the convergence of the sequence to a fixed point of many valued mapping for which the Banach fixed point theorem [11] failed. Later, in 1974 Ishikawa [12] devised a new iterative scheme known as two-step iterative scheme to establish the convergence of Lipschitzian pscudocontractive map when Mann iterative scheme failed to converge. M. A. Noor $[8,9]$ introduced and analyzed Noor iterative scheme known as three-step iterative scheme to study the approximate solutions of variational inclusions (inequalities) in Hilbert spaces by using the techniques of updating the solution and the auxiliary principle.

The data dependence abounds in literature of fixed point theory when dealing with Picard iterative scheme, but is quasi-inexistent when dealing with Mann-Ishikawa iterative scheme. In a paper of S.M. Soltuz [14] established a data dependence result concerning Mann-Ishikawa iterative scheme. There, he established the data dependence result of Ishikawa iterative scheme for contraction mappings. In [15] S.M. Soltuz and Teodor Grosan again established a data dependence result of Ishikawa iterative scheme, but there they used contractive-like operators replacing contraction mappings. Usually, Noor iterative scheme is more complicated but nevertheless more stable as Mann iterative scheme or Ishikawa iterative scheme. It is clear from literature that in which Mann or Ishikawa iterative scheme does not converge while Noor iterative scheme does. For this reason we have considered Noor iterative scheme and established a data dependence result of Noor iterative scheme for contractive-like operators. Actually, the main purpose of our paper is to establish a data dependence result of Noor iterative scheme. 


\section{Preliminaries}

In this section, we have discussed some definitions and a lemma which are important to us for representing our main result in the later sections.

Let $B$ be a real Banach space and $X$ be a nonempty convex closed and bounded subset of $B$. Let $T, S: X \rightarrow X$ be two maps. For given $x_{0}, u_{0} \in X$, we consider the Noor iterative scheme $[8,9]$ for $T$ and $S$ respectively as follows:

$$
\left.\begin{array}{ll}
x_{n+1}=\left(1-a_{n}\right) x_{n}+a_{n} T y_{n} & \\
y_{n}=\left(1-b_{n}\right) x_{n}+b_{n} T z_{n} & \\
z_{n}=\left(1-c_{n}\right) x_{n}+c_{n} T x_{n}, & \\
u_{n+1}=\left(1-a_{n}\right) u_{n}+a_{n} S v_{n} & \\
v_{n}=\left(1-b_{n}\right) u_{n}+b_{n} S w_{n} & \\
w_{n}=\left(1-c_{n}\right) u_{n}+c_{n} S u_{n}, 1,2, \ldots . .
\end{array}\right\}
$$

where the sequences $\left\{a_{n}\right\}_{n=0}^{\infty},\left\{b_{n}\right\}_{n=0}^{\infty},\left\{c_{n}\right\}_{n=0}^{\infty} \subset[0,1]$ are convergent, such that

$$
\lim _{n \rightarrow \infty} a_{n}=0, \lim _{n \rightarrow \infty} b_{n}=0, \lim _{n \rightarrow \infty} c_{n}=0 \text { and } \sum_{n=1}^{\infty} a_{n}=\infty
$$

If we put $c_{n}=b_{n}=0, \forall n \in N$ (set of all natural numbers), in the above equations then we get Mann iterative scheme [18] and if we put $c_{n}=0, \forall n \in N$ (set of all natural numbers), in the above equations then we get Ishikawa iterative scheme [12].

The map $T$ is called Kannan mappings [10], if there exists $\beta \in(0,1 / 2)$ such that for all $x, y \in X$,

$$
\|T x-T y\| \leq \beta(\|x-T x\|+\|y-T y\|)
$$

Similar mappings are Chatterjea mappings [13], for which there exists $\gamma \in(0,1 / 2)$ such that for all $x, y \in X$

$$
\|T x-T y\| \leq \gamma(\|x-T y\|+\|y-T x\|)
$$

In [16] T. Zamfirescu collected these classes and introduced the following definition: Definition 2.1 $[1,16]$. The operator $T: X \rightarrow X$ is called a Zamfirescu operator if it satisfies the condition $\mathbf{Z}$ (Zamfirescu condition) i.e., if and only if there exist the real numbers $\alpha, \beta, \gamma$ satisfying $0<\alpha<1,0<\beta, \gamma<1 / 2$ such that for each pair $x, y \in X$, at least one of the following three conditions is true: 


$$
\left.\begin{array}{ll}
\left(z_{1}\right) & \|T x-T y\| \leq \alpha\|x-y\| ; \\
\left(z_{2}\right) & \|T x-T y\| \leq \beta(\|x-T x\|+\|y-T y\|) ; \\
\left(z_{3}\right) & \|T x-T y\| \leq \gamma(\|x-T y\|+\|y-T x\|) .
\end{array}\right\}
$$

In [2] B. E. Rhoades proved that $\left(z_{1}\right),\left(z_{2}\right)$ and $\left(z_{3}\right)$ are independent conditions.

Consider $x, y \in X$. Since $T$ is a Zamfirescu operator, therefore, at least one of the conditions $\left(z_{1}\right),\left(z_{2}\right)$ and $\left(z_{3}\right)$ is satisfied by $T$. If $\left(z_{2}\right)$ holds, then

$$
\begin{aligned}
& \|T x-T y\| \leq \beta(\|x-T x\|+\|y-T y\|) \\
& \quad \leq \beta(\|x-T x\|+(\|y-x\|+\|x-T x\|+\|T x-T y\|)) \\
& \Rightarrow\|T x-T y\| \leq \frac{\beta}{(1-\beta)}\|x-y\|+\frac{2 \beta}{(1-\beta)}\|x-T x\|
\end{aligned}
$$

If $\left(z_{3}\right)$ holds, then similarly we obtain

$$
\|T x-T y\| \leq \frac{\gamma}{(1-\gamma)}\|x-y\|+\frac{2 \gamma}{(1-\gamma)}\|x-T x\|
$$

Let us denote $\lambda=\max \left\{\alpha, \frac{\beta}{(1-\beta)}, \frac{\gamma}{(1-\gamma)}\right\}$

Then we have, $0 \leq \lambda<1$ and in view of $\left(z_{1}\right)$, (2.7) and (2.8) we get the following inequality

$$
\|T x-T y\| \leq \lambda\|x-y\|+2 \lambda\|x-T x\| \text { holds } \forall x, y \in X .
$$

Formula (2.10) was obtained as in [17].

Osilike and Udomene introduced in [7] a more general definition of a quasi-contractive operator, they considered the operator for which there exists $L \geq 0$ and $q \in(0,1)$ such that

$$
\|T x-T y\| \leq q\|x-y\|+L\|x-T x\|, \quad \forall x, y \in X .
$$

Imoru and Olatinwo considered in [4], the following general definition. They are failed to name them. But in [15] S. M. Soltuz and Teodor Grosan used as contractive-like operators.

Definition 2.2 [15]. The operator $T$ is called contractive-like operator if there exist a constant $q \in(0,1)$ and a strictly increasing and continuous function $\phi:[0, \infty) \rightarrow[0, \infty)$ with $\phi(0)=0$ such that for each $x, y \in X$

$$
\|T x-T y\| \leq q\|x-y\|+\phi(\|x-T x\|), \quad \forall x, y \in X .
$$


The inequality $(1-x) \leq \exp (x), \forall x \geq 0$ leads the following remark.

Remark 2.3. Let $\left\{d_{n}\right\}$ be a nonnegative sequence such that $d_{n} \in(0,1], \forall n \in \mathbf{N}$ (Set of natural numbers). If $\sum_{n=1}^{\infty} d_{n}=\infty$, then $\prod_{n=1}^{\infty}\left(1-d_{n}\right)=0$.

Proof of remark 2.3. According to our assumption, we suppose that

$$
d_{1}=1, d_{2}=\frac{1}{2}, d_{3}=\frac{1}{3}, d_{4}=\frac{1}{4}, \ldots \ldots, d_{n}=\frac{1}{n}, \ldots \ldots \ldots
$$

That is our given sequence is

$$
\left\{d_{n}\right\}=\left\{1, \frac{1}{2}, \frac{1}{3}, \frac{1}{4}, \ldots \ldots ., \frac{1}{n}, \ldots \ldots .\right\}
$$

Hence, it is clear that

$$
\sum_{n=1}^{\infty} d_{n}=1+\frac{1}{2}+\frac{1}{3}+\frac{1}{4}+\ldots \ldots . .+\frac{1}{n}+\ldots \ldots=\infty .
$$

Now, we have to show that $\prod_{n=1}^{\infty}\left(1-d_{n}\right)=0$.

We have

$$
\prod_{n=1}^{\infty}\left(1-d_{n}\right)=\left(1-d_{1}\right) \cdot\left(1-d_{2}\right) \cdot\left(1-d_{3}\right) \cdot\left(1-d_{4}\right) \ldots \ldots \ldots . .
$$

But according to our assumption one of the values of $d_{1}, d_{2}, d_{3}, d_{4}, \ldots .$. must be equal to 1 .

Therefore, $\left(1-d_{1}\right) .\left(1-d_{2}\right) .\left(1-d_{3}\right) .\left(1-d_{4}\right) \ldots \ldots \ldots \infty=0$.

This implies that $\prod_{n=1}^{\infty}\left(1-d_{n}\right)=0$.

This completes our proof.

The following is similar to lemma from [6].

Lemma 2.4 [6]. Let $\left\{x_{n}\right\}$ be a nonnegative sequence for which one supposes there exists $n_{0} \in \mathbf{N}$, such that for all $n \geq n_{0}$ one has satisfied the following inequality:

$$
x_{n+1} \leq\left(1-\delta_{n}\right) x_{n}+\delta_{n} \sigma_{n}
$$

where $\delta_{n} \in(0,1), \forall n \in \mathbf{N}, \sum_{n=1}^{\infty} \delta_{n}=\infty$, and $\sigma_{n} \geq 0 \forall n \in \mathbf{N}$. Then, 


$$
0 \leq \lim _{n \rightarrow \infty} \sup x_{n} \leq \lim _{n \rightarrow \infty} \sup \sigma_{n} .
$$

Proof. There exists $n_{1} \in \mathbf{N}$ such that $\sigma_{n} \leq \lim _{n \rightarrow \infty} \sup \sigma_{n}, \forall n \geq n_{1}$. Set $n_{2}=\left\{n_{0}, n_{1}\right\}$ such that the following inequality holds, for all $n \geq n_{1}$ :

$$
x_{n+1} \leq\left(1-\delta_{n}\right)\left(1-\delta_{n-1}\right) \ldots \ldots\left(1-\delta_{n_{1}}\right) x_{n_{1}}+\lim _{n \rightarrow \infty} \sup \sigma_{n}
$$

Using the above Remark 2.3 with $d_{n}=\delta_{n}$, we get the conclusion. In order to prove (2.15), consider (2.13) and the induction step:

$$
\begin{aligned}
x_{n+2} & \leq\left(1-\delta_{n+1}\right) x_{n+1}+\delta_{n+1} \sigma_{n+1} \\
& \leq\left(1-\delta_{n+1}\right)\left(1-\delta_{n}\right)\left(1-\delta_{n-1}\right) \ldots . .\left(1-\delta_{n_{1}}\right) x_{n_{1}}+\left(1-\delta_{n+1}\right) \limsup _{n \rightarrow \infty}+\delta_{n+1} \sigma_{n+1} \\
& =\left(1-\delta_{n+1}\right)\left(1-\delta_{n}\right)\left(1-\delta_{n-1}\right) \ldots \ldots\left(1-\delta_{n_{1}}\right) x_{n_{1}}+\left(1-\delta_{n+1}\right) \lim _{n \rightarrow \infty} \sup \sigma_{n} .
\end{aligned}
$$

This completes our proof.

\section{Main Results}

In this section, we have stated and proved our main results.

Theorem 3.1. Let $B$ be a real Banach space, $X \subset B$ a nonempty, closed and convex set, and $T: X \rightarrow X$ a contractive-like map with $m^{*}$ being the fixed point. Then for all $x_{0} \in X$, the Noor iterative scheme defined by the equation (2.1) converges to the unique fixed point of $T$.

Proof. First we prove the uniqueness of the fixed point of $T$. If possible let the mapping $T$ has two distinct fixed points $m^{*}$ and $n^{*}$. Then by using the definition 2.2 we get

$$
\left\|m^{*}-n^{*}\right\|=\left\|T m^{*}-T n^{*}\right\| \leq q\left\|m^{*}-n^{*}\right\|+\phi\left(\left\|m^{*}-T m^{*}\right\|\right)=q\left\|m^{*}-n^{*}\right\|
$$

This implies that $m^{*}=n^{*}$. Hence the fixed point of $T$ is unique.

Now, from (2.1) and (2.12) we obtain 


$$
\begin{aligned}
\left\|x_{n+1}-m^{*}\right\|= & \left\|\left(1-a_{n}\right) x_{n}+a_{n} T y_{n}-m^{*}\right\| \\
\leq & \left(1-a_{n}\right)\left\|x_{n}-m^{*}\right\|+a_{n}\left\|T y_{n}-T m^{*}\right\| \\
\leq & \left(1-a_{n}\right)\left\|x_{n}-m^{*}\right\|+q a_{n}\left\|y_{n}-m^{*}\right\| \\
\leq & \left(1-a_{n}\right)\left\|x_{n}-m^{*}\right\|+q a_{n}\left(1-b_{n}\right)\left\|x_{n}-m^{*}\right\|+q a_{n} b_{n}\left\|T z_{n}-T m^{*}\right\| \\
\leq & \left(1-a_{n}\right)\left\|x_{n}-m^{*}\right\|+q a_{n}\left(1-b_{n}\right)\left\|x_{n}-m^{*}\right\|+q^{2} a_{n} b_{n}\left\|z_{n}-m^{*}\right\| \\
\leq & \left(1-a_{n}\right)\left\|x_{n}-m^{*}\right\|+q a_{n}\left(1-b_{n}\right)\left\|x_{n}-m^{*}\right\|+q^{2} a_{n} b_{n}\left(1-c_{n}\right)\left\|x_{n}-m^{*}\right\|+q^{2} a_{n} b_{n} c_{n}\left\|T x_{n}-T m^{*}\right\| \\
\leq & \left(1-a_{n}\right)\left\|x_{n}-m^{*}\right\|+q a_{n}\left(1-b_{n}\right)\left\|x_{n}-m^{*}\right\|+q^{2} a_{n} b_{n}\left(1-c_{n}\right)\left\|x_{n}-m^{*}\right\|+q^{3} a_{n} b_{n} c_{n}\left\|x_{n}-m^{*}\right\| \\
= & \left(\left(1-a_{n}\right)+q a_{n}\left(1-b_{n}\right)+q^{2} a_{n} b_{n}\left(1-c_{n}\right)+q^{3} a_{n} b_{n} c_{n}\right)\left\|x_{n}-m^{*}\right\| \\
= & \left(1-a_{n}\left(1-q\left(1-b_{n}\right)-q^{2} b_{n}\left(1-c_{n}\right)-q^{3} b_{n} c_{n}\right)\right)\left\|x_{n}-m^{*}\right\| \\
& =\left(1-a_{n}\left(1-q\left(1-b_{n}+q b_{n}\left(1-c_{n}\right)+q^{2} b_{n} c_{n}\right)\right)\right)\left\|x_{n}-m^{*}\right\| \\
& =\left(1-a_{n}\left(1-q\left(1-b_{n}\left(1-q\left(1-c_{n}\right)-q^{2} c_{n}\right)\right)\right)\right)\left\|x_{n}-m^{*}\right\| \\
& =\left(1-a_{n}\left(1-q\left(1-b_{n}\left(1-q\left(1-c_{n}+q c_{n}\right)\right)\right)\right)\right)\left\|x_{n}-m^{*}\right\| \\
& =\left(1-a_{n}\left(1-q\left(1-b_{n}\left(1-q\left(1-c_{n}\left(1-q_{n}\right)\right)\right)\right)\right)\right)\left\|x_{n}-m^{*}\right\| . \\
\text { i.e. } . & \left\|x_{n+1}-m^{*}\right\| \leq\left(1-a_{n}\left(1-q\left(1-b_{n}\left(1-q\left(1-c_{n}\left(1-q_{n}\right)\right)\right)\right)\right)\right)\left\|x_{n}-m^{*}\right\| .
\end{aligned}
$$

But, according to our supposition we can write

$$
\left(1-a_{n}\left(1-q\left(1-b_{n}\left(1-q\left(1-c_{n}\left(1-q_{n}\right)\right)\right)\right)\right)\right) \leq\left(1-a_{n}(1-q)\right) .
$$

Combining (3.2) and (3.1) we get

$$
\begin{aligned}
\left\|x_{n+1}-m^{*}\right\| & \leq\left(1-a_{n}(1-q)\right)\left\|x_{n}-m^{*}\right\| \\
& \leq\left(1-a_{n}(1-q)\right)\left(1-a_{n-1}(1-q)\right)\left\|x_{n-1}-m^{*}\right\| \\
& \leq\left(1-a_{n}(1-q)\right)\left(1-a_{n-1}(1-q)\right)\left(1-a_{n-2}(1-q)\right)\left\|x_{n-2}-m^{*}\right\| \\
& \leq \ldots \leq\left[\prod_{k=0}^{n}\left(1-a_{k}(1-q)\right)\right]\left\|x_{0}-m^{*}\right\|
\end{aligned}
$$

Since, $\sum_{n=1}^{\infty} a_{n}=\infty$ implies $\sum_{n=1}^{\infty} a_{n}(1-q)=\infty \forall q \in(0,1)$. So, by the Remark 2.3 we can write,

$$
\lim _{n \rightarrow \infty}\left[\prod_{k=0}^{n}\left(1-a_{k}(1-q)\right)\right]=0
$$

From (3.4) and (3.5) we get 


$$
\begin{aligned}
& \lim _{n \rightarrow \infty}\left\|x_{n+1}-m^{*}\right\|=0 \\
& \therefore \lim _{n \rightarrow \infty}\left\|x_{n}-m^{*}\right\|=0
\end{aligned}
$$

This completes our proof.

Theorem 3.2. Let $B$ be a real Banach space, $X \subset B$ a nonempty, closed and convex set, and let $\varepsilon>0$ be a fixed number. If $T: X \rightarrow X$ is a contractive-like operator with the fixed point $x^{*}$ and $S: X \rightarrow X$ is an operator with the fixed point $u^{*}$, (supposed nearest to $x^{*}$ ), and if the following relation is satisfied:

$$
\|T z-S z\| \leq \varepsilon, \forall z \in X
$$

Then

$$
\left\|x^{*}-u^{*}\right\| \leq \frac{\varepsilon}{(1-q)}, \quad \forall q \in(0,1) .
$$

Proof. From (2.1) and (2.2), we get

$$
x_{n+1}-u_{n+1}=\left(1-a_{n}\right)\left(x_{n}-u_{n}\right)+a_{n}\left(T y_{n}-S v_{n}\right)
$$

Now, taking norm on both sides of (3.8) we have

$$
\begin{aligned}
\left\|x_{n+1}-u_{n+1}\right\| & =\left\|\left(1-a_{n}\right)\left(x_{n}-u_{n}\right)+a_{n}\left(T y_{n}-S v_{n}\right)\right\| \\
& \leq\left(1-a_{n}\right)\left\|x_{n}-u_{n}\right\|+a_{n}\left\|S v_{n}-T y_{n}\right\| \\
& \leq\left(1-a_{n}\right)\left\|x_{n}-u_{n}\right\|+a_{n}\left\|S v_{n}-T v_{n}+T v_{n}-T y_{n}\right\| \\
& \leq\left(1-a_{n}\right)\left\|x_{n}-u_{n}\right\|+a_{n}\left\|T v_{n}-S v_{n}\right\|+a_{n}\left\|T v_{n}-T y_{n}\right\|
\end{aligned}
$$




$$
\begin{aligned}
& \leq\left(1-a_{n}\right)\left\|x_{n}-u_{n}\right\|+a_{n} \varepsilon+q a_{n}\left\|y_{n}-v_{n}\right\|+a_{n} \phi\left(\left\|y_{n}-T y_{n}\right\|\right) \\
& \leq\left(1-a_{n}\right)\left\|x_{n}-u_{n}\right\|+a_{n} \varepsilon+q a_{n}\left(1-b_{n}\right)\left\|x_{n}-u_{n}\right\|+q a_{n} b_{n}\left\|T z_{n}-S w_{n}\right\|+a_{n} \phi\left(\left\|y_{n}-T y_{n}\right\|\right) \\
& \leq\left(1-a_{n}\right)\left\|x_{n}-u_{n}\right\|+a_{n} \varepsilon+q a_{n}\left(1-b_{n}\right)\left\|x_{n}-u_{n}\right\| \\
& +q a_{n} b_{n}\left\|S w_{n}-T w_{n}+T w_{n}-T z_{n}\right\|+a_{n} \phi\left(\left\|y_{n}-T y_{n}\right\|\right) \\
& \leq\left(1-a_{n}\right)\left\|x_{n}-u_{n}\right\|+a_{n} \varepsilon+q a_{n}\left(1-b_{n}\right)\left\|x_{n}-u_{n}\right\|+q a_{n} b_{n} \varepsilon \\
& +q a_{n} b_{n}\left\|T z_{n}-T w_{n}\right\|+a_{n} \phi\left(\left\|y_{n}-T y_{n}\right\|\right) \\
& \leq\left(1-a_{n}\right)\left\|x_{n}-u_{n}\right\|+a_{n} \varepsilon+q a_{n}\left(1-b_{n}\right)\left\|x_{n}-u_{n}\right\|+q a_{n} b_{n} \varepsilon \\
& +q^{2} a_{n} b_{n}\left\|z_{n}-w_{n}\right\|+q a_{n} b_{n} \phi\left(\left\|z_{n}-T z_{n}\right\|\right)+a_{n} \phi\left(\left\|y_{n}-T y_{n}\right\|\right) \\
& \leq\left(1-a_{n}\right)\left\|x_{n}-u_{n}\right\|+a_{n} \varepsilon+q a_{n}\left(1-b_{n}\right)\left\|x_{n}-u_{n}\right\|+q a_{n} b_{n} \varepsilon+q^{2} a_{n} b_{n}\left(1-c_{n}\right)\left\|x_{n}-u_{n}\right\| \\
& +q^{2} a_{n} b_{n} c_{n}\left\|T x_{n}-S u_{n}\right\|+q a_{n} b_{n} \phi\left(\left\|z_{n}-T z_{n}\right\|\right)+a_{n} \phi\left(\left\|y_{n}-T y_{n}\right\|\right) \\
& \leq\left(1-a_{n}\right)\left\|x_{n}-u_{n}\right\|+a_{n} \varepsilon+q a_{n}\left(1-b_{n}\right)\left\|x_{n}-u_{n}\right\|+q a_{n} b_{n} \varepsilon+q^{2} a_{n} b_{n}\left(1-c_{n}\right)\left\|x_{n}-u_{n}\right\| \\
& +q^{2} a_{n} b_{n} c_{n}\left(\left\|T x_{n}-T u_{n}\right\|+\left\|T u_{n}-S u_{n}\right\|\right)+q a_{n} b_{n} \phi\left(\left\|z_{n}-T z_{n}\right\|\right)+a_{n} \phi\left(\left\|y_{n}-T y_{n}\right\|\right) \\
& \leq\left(1-a_{n}\right)\left\|x_{n}-u_{n}\right\|+a_{n} \varepsilon+q a_{n}\left(1-b_{n}\right)\left\|x_{n}-u_{n}\right\|+q a_{n} b_{n} \varepsilon+q^{2} a_{n} b_{n}\left(1-c_{n}\right)\left\|x_{n}-u_{n}\right\| \\
& +q^{2} a_{n} b_{n} c_{n} \varepsilon+q^{2} a_{n} b_{n} c_{n}\left\|T x_{n}-T u_{n}\right\|+q a_{n} b_{n} \phi\left(\left\|z_{n}-T z_{n}\right\|\right)+a_{n} \phi\left(\left\|y_{n}-T y_{n}\right\|\right) \\
& \leq\left(1-a_{n}\right)\left\|x_{n}-u_{n}\right\|+a_{n} \varepsilon+q a_{n}\left(1-b_{n}\right)\left\|x_{n}-u_{n}\right\|+q a_{n} b_{n} \varepsilon+q^{2} a_{n} b_{n}\left(1-c_{n}\right)\left\|x_{n}-u_{n}\right\| \\
& +q^{2} a_{n} b_{n} c_{n} \varepsilon+q^{3} a_{n} b_{n} c_{n}\left\|x_{n}-u_{n}\right\|+q^{2} a_{n} b_{n} c_{n} \phi\left(\left\|x_{n}-T x_{n}\right\|\right) \\
& +q a_{n} b_{n} \phi\left(\left\|z_{n}-T z_{n}\right\|\right)+a_{n} \phi\left(\left\|y_{n}-T y_{n}\right\|\right) \\
& =\left(1-a_{n}+q a_{n}\left(1-b_{n}\right)+q^{2} a_{n} b_{n}\left(1-c_{n}\right)+q^{3} a_{n} b_{n} c_{n}\right)\left\|x_{n}-u_{n}\right\|+a_{n} \varepsilon+q a_{n} b_{n} \varepsilon \\
& +q^{2} a_{n} b_{n} c_{n} \varepsilon+q^{2} a_{n} b_{n} c_{n} \phi\left(\left\|x_{n}-T x_{n}\right\|\right)+q a_{n} b_{n} \phi\left(\left\|z_{n}-T z_{n}\right\|\right)+a_{n} \phi\left(\left\|y_{n}-T y_{n}\right\|\right) \\
& =\left(1-a_{n}\left(1-q\left(1-b_{n}\left(1-q\left(1-c_{n}\left(1-q_{n}\right)\right)\right)\right)\right)\right)\left\|x_{n}-u_{n}\right\|+q^{2} a_{n} b_{n} c_{n} \phi\left(\left\|x_{n}-T x_{n}\right\|\right) \\
& +q a_{n} b_{n} \phi\left(\left\|z_{n}-T z_{n}\right\|\right)+a_{n} \phi\left(\left\|y_{n}-T y_{n}\right\|\right)+a_{n} \varepsilon+q a_{n} b_{n} \varepsilon+q^{2} a_{n} b_{n} c_{n} \varepsilon \\
& \leq\left(1-a_{n}(1-q)\right)\left\|x_{n}-u_{n}\right\|+a_{n}\left(q^{2} b_{n} c_{n} \phi\left(\left\|x_{n}-T x_{n}\right\|\right)+q b_{n} \phi\left(\left\|z_{n}-T z_{n}\right\|\right)\right. \\
& \left.+\phi\left(\left\|y_{n}-T y_{n}\right\|\right)+\varepsilon+q b_{n} \varepsilon+q^{2} b_{n} c_{n} \varepsilon\right) \\
& \left(q^{2} b_{n} c_{n} \phi\left(\left\|x_{n}-T x_{n}\right\|\right)+q b_{n} \phi\left(\left\|z_{n}-T z_{n}\right\|\right)\right. \\
& =\left(1-a_{n}(1-q)\right)\left\|x_{n}-u_{n}\right\|+\left(a_{n}(1-q)\right) \frac{\left.+\phi\left(\left\|y_{n}-T y_{n}\right\|\right)+\varepsilon+q b_{n} \varepsilon+q^{2} b_{n} c_{n} \varepsilon\right)}{(1-q)} \\
& \left(q^{2} b_{n} c_{n} \phi\left(\left\|x_{n}-T x_{n}\right\|\right)+q b_{n} \phi\left(\left\|z_{n}-T z_{n}\right\|\right)\right. \\
& \left\|x_{n+1}-u_{n+1}\right\| \leq\left(1-a_{n}(1-q)\right)\left\|x_{n}-u_{n}\right\|+\left(a_{n}(1-q)\right) \frac{\left.+\phi\left(\left\|y_{n}-T y_{n}\right\|\right)+\varepsilon+q b_{n} \varepsilon+q^{2} b_{n} c_{n} \varepsilon\right)}{(1-q)} \text {. }
\end{aligned}
$$

It is clear that $\lim _{n \rightarrow \infty} \phi\left(\left\|x_{n}-T x_{n}\right\|\right)=\lim _{n \rightarrow \infty} \phi\left(\left\|y_{n}-T y_{n}\right\|\right)=\lim _{n \rightarrow \infty} \phi\left(\left\|z_{n}-T z_{n}\right\|\right)=0$ because $\phi$ is a continuous function and $\left\{x_{n}\right\},\left\{y_{n}\right\}$ and $\left\{z_{n}\right\}$ all are converge to the fixed point of $T$. 
Now, if we put

$$
\begin{aligned}
\delta_{n}= & a_{n}(1-q) \\
& \left(q^{2} b_{n} c_{n} \phi\left(\left\|x_{n}-T x_{n}\right\|\right)+q b_{n} \phi\left(\left\|z_{n}-T z_{n}\right\|\right)\right. \\
\sigma_{n}= & \left.+\phi\left(\left\|y_{n}-T y_{n}\right\|\right)+\varepsilon+q b_{n} \varepsilon+q^{2} b_{n} c_{n} \varepsilon\right)
\end{aligned} .
$$

Then, from the Lemma 2.4 we get

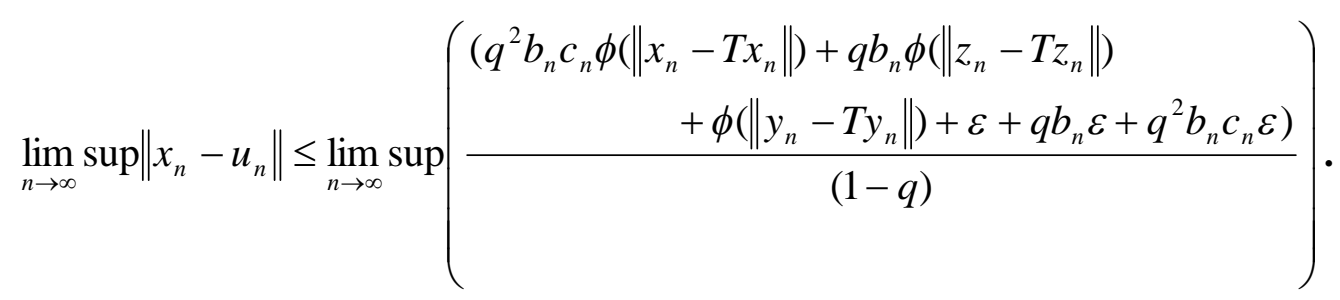

This implies that

$$
\left\|x^{*}-u^{*}\right\| \leq \frac{\varepsilon}{(1-q)} .
$$

This completes our proof.

Remark 3.3. (i) If we put $c_{n}=0, \forall n \in \mathbf{N}$, then we obtain the data dependence for Ishikawa iterative scheme.

(ii) If we put $c_{n}=b_{n}=0, \forall n \in \mathbf{N}$, then we obtain the data dependence for Mann iterative scheme.

(iii) The Zamfirescu operator and implicitly (Chatterjea and Kannan) are contractive-like operator, therefore our Theorem 3.2 remains true for all of these classes.

Proof. From, the definition of Noor iterative scheme we have, for given $x_{0} \in X$

$$
\left.\begin{array}{ll}
x_{n+1}=\left(1-a_{n}\right) x_{n}+a_{n} T y_{n} & \\
y_{n}=\left(1-b_{n}\right) x_{n}+b_{n} T z_{n} & \\
z_{n}=\left(1-c_{n}\right) x_{n}+c_{n} T x_{n}, & n=0,1,2, \ldots . .
\end{array}\right\}
$$

where the sequences $\left\{a_{n}\right\}_{n=0}^{\infty},\left\{b_{n}\right\}_{n=0}^{\infty},\left\{c_{n}\right\}_{n=0}^{\infty} \subset[0,1]$ are convergent, such that

$$
\lim _{n \rightarrow \infty} a_{n}=0, \lim _{n \rightarrow \infty} b_{n}=0, \lim _{n \rightarrow \infty} c_{n}=0 \text { and } \sum_{n=1}^{\infty} a_{n}=\infty
$$

Now, if we put $c_{n}=0, \forall n \in \mathbf{N}$ and $c_{n}=b_{n}=0, \forall n \in \mathbf{N}$ in the above equation, then 
we obtain the Ishikawa iterative scheme

$$
\begin{aligned}
& x_{n+1}=\left(1-a_{n}\right) x_{n}+a_{n} T y_{n} \\
& \left.y_{n}=\left(1-b_{n}\right) x_{n}+b_{n} T z_{n} \quad n=0,1,2, \ldots . .\right\}
\end{aligned}
$$

where the sequences $\left\{a_{n}\right\}_{n=0}^{\infty},\left\{b_{n}\right\}_{n=0}^{\infty} \subset[0,1]$ are convergent, such that

$$
\lim _{n \rightarrow \infty} a_{n}=0, \lim _{n \rightarrow \infty} b_{n}=0 \text { and } \sum_{n=1}^{\infty} a_{n}=\infty
$$

and the Mann Iterative scheme $\left.x_{n+1}=\left(1-a_{n}\right) x_{n}+a_{n} T y_{n} \quad n=0,1,2, \ldots ..\right\}$ where the sequences $\left\{a_{n}\right\}_{n=0}^{\infty} \subset[0,1]$ are convergent, such that $\lim _{n \rightarrow \infty} a_{n}=0$ and $\sum_{n=1}^{\infty} a_{n}=\infty$ respectively.

Now, it is easily verified that, if we replace the Noor iterative scheme by Ishikawa iterative scheme and Mann Iterative scheme in our theorem 3.1, then we obtain the data dependence for Ishikawa iterative scheme and Mann Iterative scheme respectively.

This proves (i) and (ii).

To prove (iii), we observe the equations (2.4), (2.5), (2.6) and (2.12) and from this observation we can comment that, the contractive-like operator is a general operator among the operator of Zamfirescu, Chatterjea, Kannan and contractive-like. Since, our theorem 3.2 is true for the general operator, contractive-like operator. Hence, it will be must true for these entire operator.

This completes our proof.

\section{Numerical Example}

In this section, we have given an example to realize our Theorem 3.2, which is follows from [15] and [17].

Example 4.1 Let $T: \mathrm{R} \rightarrow \mathrm{R}$ be a mapping defined by

$$
T x=\left\{\begin{array}{cc}
1 ; & \text { when } x \in(-\infty, 3] \\
-1.5 ; & \text { when } x \in(3,+\infty) .
\end{array}\right.
$$

Where, $\mathrm{R}$ denotes the set of real numbers. Then, it is clear that $T$ is contractive-like operator with $q=0.1$ and $\phi$ is an identity function and $x=1$ is the unique fixed point of $T$.

Now, we consider an arbitrary mapping $S: \mathrm{R} \rightarrow \mathrm{R}$, which is defined as follows

$$
S x=\left\{\begin{array}{cc}
2 ; & \text { when } x \in(-\infty, 3] \\
-2.5 ; & \text { when } x \in(3,+\infty) .
\end{array}\right.
$$

It is clear that $x=2$ is the unique fixed point of $S$. Now, we cheek it by applying Noor iterative scheme and form an estimate by applying our Theorem 3.2. 
If we take the initial approximation as $x_{0}=u_{0}=1$ and $a_{n}=b_{n}=c_{n}=1 /(n+1)$, then for $S$ the Noor iterative scheme gives us the following result:

\section{Iteration $\operatorname{Step}(n)$}

$$
\begin{gathered}
n=1 \\
\ldots \ldots . \\
n=5 \\
\ldots \ldots \ldots \\
n=10 \\
\ldots \ldots \ldots . . \\
n=100 \\
\ldots \ldots \ldots \ldots \\
\ldots \ldots \ldots \ldots \\
n \rightarrow \infty
\end{gathered}
$$

\section{Obtained value by Noor iterative scheme}

1.96875

1.9990234375

1.9999999999999999999999999999992

The above computations can be obtained also by using a Matlab-7 program. This leads us to conclude that Noor iterative scheme applied to $S$ converges to fixed point $x=2$, which is the unique fixed point of $S$.

Take $\varepsilon$ to be the distance between the two maps as follows:

$$
\|S x-T x\| \leq 1, \forall x \in \mathrm{R} \text {, i.e., } \varepsilon=1 \text {. }
$$

Eventually, one can see that the distance between the fixed points of $T$ and $S$ is 1 . Actually, without knowing the fixed point of $S$ and without computing it, via Theorem 3.2 , we can formulate the following estimate for it:

$$
\left\|x^{*}-u^{*}\right\| \leq \frac{1}{(1-0.1)}=\frac{1}{0.9}=1.11111111 \text {. }
$$

That is the distance between the fixed points of $T$ and $S$ must be less than 1.11111111 .

\section{Conclusion}

Our result is an extension and improvement of the result of S. M. Soltuz and Teodor Grosan [15]. Here, we have established the data dependence of more general Noor iterative scheme. By applying our Theorem 3.2 we can estimate the unknown fixed point of an arbitrary mapping without computing fixed point of it. The data dependence of Noor iterative scheme is general comparing with the data dependence of Mann and Ishikawa iterative schemes, because from the data dependence of Noor iterative scheme we can easily obtain the data dependence of Mann and Ishikawa iterative schemes. 


\section{REFERENCES}

[1] B. E. Rhoades, Fixed point iterations using infinite matrices, Transactions of the American Mathematical Society, vol. 196, pp. 161-176, 1974.

[2] B. E. Rhoades, A comparison of various definitions of contractive mappings, Transactions of the American Mathematical Society, vol. 226, pp. 257-290, 1977.

[3] C. E. Chidume and S. A. Mutangadura, An example of the Mann iteration method for Lipschitz Pseudocontractions, Proceedings of the American Mathematical Society, vol. 129, no. 8, pp. 2359-2363, 2001.

[4] C. O. Imoru and M. O. Olatinwo, On the stability of Picard and Mann iteration processes, Carpathian Journal of Mathematics, vol. 19, no. 2, pp. 155-160, 2003.

[5] D. R. Smart, Fixed point theorems, Cambridge University Press, 1974.

[6] J. A. Park, Mann-iteration process for the fixed point of strictly pseudo-contractive mapping in some Banach spaces, Journal of the Korean Mathematical Society, vol. 31, no. 3, pp. 333-337, 1994.

[7] M. O. Osilike and A. Udomene, Short proofs of stability results for fixed point iteration procedures for a class of contractive-type mappings, Indian Journal of Pure and Applied Mathematics, vol. 30, no. 12, pp. 1229-1234, 1999.

[8] M. A. Noor, New approximation schemes for general variational inequalities, J. Math. Anal. Appl., vol. 251, pp 217-229, 2000.

[9] M. A. Noor, Three-step iterative algorithms for multivalued variational inclusions, J. Math. Anal. Appl., vol. 255, pp 589-604, 2001.

[10] R. Kannan, Some results on fixed points, Bulletin of the Calcutta Mathematical Society, vol.60, pp.71-76, 1968.

[11] S. Banach, Sur les operations dans les ensembles assembles abstraits et leur Applications aux equations integrals, Fund. Math. 3, pp 133-181, 1922.

[12] S. Ishikawa, Fixed points by a new iteration method, Proc. Amer. Math. Soc. 44, pp 147150, 1974.

[13] S. K. Chatterjea, Fixed-point theorems, Comptes Rendus de l'Acad'emie Bulgare des Sciences, vol. 25, pp.727-730, 1972.

[14] S. M. Soltuz, Data dependence for Ishikawa iteration, Lecturas Matem'aticas, vol. 25, no. 2, pp. 149-155, 2004.

[15] S. M. Soltuz and Teodor Grosan, Data Dependence for Ishikawa Iteration When Dealing with Contractive-Like Operators, Fixed Point Theory and Applications, Hindawi Publishing Corporation, Article ID 242916, 7 pages, 2008.

[16] T. Zamfirescu, Fix point theorems in metric spaces, Archiv der Mathematik, vol. 23, no. 1, pp. 292-298, 1972.

[17] V. Berinde, On the convergence of the Ishikawa iteration in the class of quasi contractive operators, Acta Mathematica Universitatis Comenianae, vol. 73, no. 1, pp. 119-126, 2004.

[18] W. R. Mann, Mean value methods in iteration, Proc. Amer. Math. Soc. 4, pp 506-510, 1953. 